\title{
A note on log-optimal portfolios in exponential Lévy markets
}

\author{
T. R. Hurd* \\ Dept. of Math and Stats \\ McMaster University \\ Hamilton, Ontario \\ L8S 4K1
}

May 21, 2004

\begin{abstract}
In this note we revisit Merton's optimal portfolio selection problem within an exponential Lévy market, for an agent acting with a canonical utility function, the logarithm. From the explicit construction of solutions, we observe features which arise related to shortselling and borrowing, not noted in earlier treatments by [12],[7],[10].
\end{abstract}

\section{Introduction}

Merton's problem [13] is the problem of portfolio selection to maximize the expected utility of wealth at a terminal time $T$. This is a classic problem of mathematical finance which has spawned a great amount of important work. For a detailed review of the history of Merton's problem, please see [11],[15] and the references therein. An interesting pathology of the general theory has been observed by, namely the possibility that some primary assets may fail to be martingales under the utility optimal pricing measure, but are rather strict supermartingales. Moreover, the pricing measure itself may fail to be a probability measure.

In the present note, we focus on a simple and natural example of this pathology which arises for the logarithmic utility in the class of exponential

\footnotetext{
${ }^{*}$ Research supported by the Natural Sciences and Engineering Research Council of Canada and MITACS, Canada
} 
Lévy market models where the log returns exhibits unbounded jumps of both signs. To this end, we apply the theory of [12] leading to explicit solutions which show three qualitatively different regimes, for different ranges of parameters. These examples illustrate the main point that when an asset is "underpriced", it will be a supermartingale under the pricing measure and not a martingale. In one regime it happens that the numeraire itself is underpriced: then the Radon-Nikodym derivative of the pricing measure is seen to be not a martingale, and the pricing measure is not a probability measure. Alternatively, in the regime where the mean rate of return of the risky asset is negative, the (discounted) risky asset is a supermartingale but not a martingale under the pricing measure.

The logarithmic utility has a long history dating back to [?] who noted the special fact that log optimal portfolios maximize long term growth. Moreover, the "growth optimal portfolio" $X_{t}^{*}$ serves as the natural numeraire, meaning that the fair price at time $t<T$ of a claim $V_{T}$ can be consistently defined by

$$
V_{t}=X_{t}^{*} E_{t}\left[V_{T} / X_{T}^{*}\right]
$$

In section 2, we place our problem within the general theory of semimartingale market models. In Proposition 2.1, we state a set of sufficient conditions for a solution of the optimal problem, which is apparently broad enough to include the economically important cases, when the domain of the utility is $(0, \infty)$. In section 3 , we analyze the logartihmic utility and give in Proposition 3.1 a general form for the solutions. The various cases illustrate some of the subtle points which arise in the presence of shortselling and borrowing constraints.

In order to keep the exposition focused on essentials, we omit technical details for which the careful reader is referred to the existing literature, notably ??, ??. Some references to utility based optimal portfolios, hedging and pricing are: [2],[6], [7],[10],[8],[4], [1],[?],[14],[?],[?]

\section{Setup}

The general semimartingale market framework is becoming quite standard [5],[12],[10],[2]. Within the filtered probability space $\left(\Omega, \mathcal{F},\left(\mathcal{F}_{t}\right)_{t \in[0, T]}, P\right)$ satisfying the "usual conditions" (right continuity and completeness), we let $\tilde{S}=\left(\tilde{S}^{0}, \ldots, \tilde{S}^{d}\right)$ be a $d+1$-dimensional càdlàg semimartingale. As numeraire we take $\tilde{S}^{0}$, which is therefore assumed to be positive $\tilde{S}_{t}^{0}>0$ a.s. The discounted price process is then $S=\tilde{S} / \tilde{S}^{0}=\left(1, S^{1}, \ldots, S^{d}\right)$. To ensure a viable market, free of arbitrage, we assume the technical condition "No Free 
Lunch with Vanishing Risk" (NFLVR), and by the fundamental theorem of asset pricing [5] this means

NFLVR assumption: $\mathcal{M}_{\sigma}:=\{$ equivalent $\sigma$-martingale measures $\} \neq \emptyset$.

For a detailed treatment of the theory of general semimartingales we use in this paper, see [9].

A general utility function $U: \mathbb{R} \rightarrow \mathbb{R} \cup\{-\infty\}$ is a differentiable monotonically increasing concave function. The domain of $U$ is the interval $(D, \infty)$ where $D=\inf \{x \mid U(x)>-\infty\}$. We work with the condition $D>-\infty$ which places us within the framework of [12]. We assume also

Inada Conditions: (i) $\lim _{x \rightarrow D^{+}} U^{\prime}(x)=\infty, \quad$ (ii) $\lim _{x \rightarrow \infty} U^{\prime}(x)=0$.

Following [12], we define the space of admissible portfolios with initial wealth $x>D$ to be

$$
\mathcal{X}_{D}(x)=\left\{\text { processes } X_{t}=x+H \cdot S_{t} \mid H \in L(S), X_{t} \geq D\right\}
$$

$\left(L(S)\right.$ denotes the space of integrands for which $H \cdot S:=\int H_{t} d S_{t}$ is defined). Note that $\mathcal{X}_{D}(x)=D+(x-D) \mathcal{X}$ where $\mathcal{X}:=\mathcal{X}_{0}(1)$. The following space is a natural "dual" to $\mathcal{X}$ :

$$
\mathcal{Y}(y)=\left\{\text { processes } Y_{t} \geq 0 \mid Y_{0}=y, X_{t} Y_{t} \text { is supermartingale } \forall X \in \mathcal{X}\right\}
$$

Note that $\mathcal{Y}(y)=y \mathcal{Y}$ where $\mathcal{Y}:=\mathcal{Y}(1)$.

Merton's problem, which we call here the "primal problem", is to solve

$$
u(x)=\sup _{X \in \mathcal{X}_{D}(x)} E U\left(X_{T}\right)
$$

for any initial wealth $x>D$, and hence determine the value function $u$ : $(D, \infty) \rightarrow \mathbb{R}$.

As is now familiar, it is helpful to introduce the dual utility function via the Legendre transform

$$
V(y)=\sup _{x \in(D, \infty)} U(x)-x y .
$$

Since $U^{\prime}$ exists, $V(y)=U\left(x^{*}\right)-x^{*} y$ for $U^{\prime}\left(x^{*}\right)=y$, provided $y \in \operatorname{Ran}\left(U^{\prime}\right)=$ $(0, \infty)$. So defined, $V:(0, \infty) \rightarrow \mathbb{R}$ is differentiable and convex. We also have the inverse transform: for $x \in(D, \infty), U(x)=\inf _{y \in(0, \infty)} V(y)+x y$.

The "dual problem" is to solve

$$
v(y)=\inf _{Y \in \mathcal{Y}(y)} E V\left(Y_{T}\right)
$$


for any initial value $y>0$, and hence determine the dual value function $v:(0, \infty) \rightarrow \mathbb{R}$. In almost all examples, the optimizer of this problem will turn out to be a martingale, and hence yields a "separating measure" or "pricing measure" $Q \ll P$ with respect to which all admissible portfolios are supermartingales. However, we will exhibit a natural example which does not give such an interpretation.

The practical essence of [12] can be understood by the following proposition which lists sufficient conditions for finding solutions of (1) and (2). The proof we give is quite elementary given Proposition 3.1 of [12]. The remaining technical difficulties of that paper are to determine a natural conditions on the utility $U$ for the existence of solutions of this type. To avoid a trivial complication, from now on we specialize to the case $D=0$.

Proposition 2.1 (Sufficient conditions) Suppose $\left(x^{*}, y^{*}, X^{*}, Y^{*}\right) \in(0, \infty)^{2} \times$ $\mathcal{X} \times \mathcal{Y}$ satisfy

$$
x^{*} X_{T}^{*}=-V^{\prime}\left(y^{*} Y_{T}^{*}\right) \text { almost surely; }
$$

$$
E X_{T}^{*} Y_{T}^{*}=1
$$

Then

1. $x^{*} X^{*}$ is optimal for the primal problem (1) with $x=x^{*}$;

2. $y^{*} Y^{*}$ is optimal for the dual problem (2) with $y=y^{*}$;

3.

$$
u\left(x^{*}\right)=\inf _{y} v(y)+x^{*} y=v\left(y^{*}\right)+x^{*} y^{*} .
$$

Remark 2.2 Theorem 2.2 of [12] also gives the following necessary conditions for a solution of the above form: $u(x)<\infty$ for some $x>0$ and $U$ satisfies the condition of reasonable elasticity $\lim _{x \rightarrow \infty} \frac{x U^{\prime}(x)}{U(x)}<1$.

Before proceeding to the elementary proof, we need to give the background to one of the main technical results of [12]. They introduce two convex spaces constructed from $\mathcal{X}$ and $\mathcal{Y}$ :

$$
\begin{aligned}
\mathcal{C} & =\left\{g \in L^{0} \mid 0 \leq g \leq X_{T} \text { for some } X \in \mathcal{X}\right\} \\
\mathcal{D} & =\left\{h \in L^{0} \mid 0 \leq h \leq Y_{T} \text { for some } Y \in \mathcal{Y}\right\}
\end{aligned}
$$


where $L^{0} \equiv L^{0}\left(\Omega, \mathcal{F}_{T}, P\right)$ denotes the space of $\mathcal{F}_{T}$ random variables. Then Proposition 3.1 of [12] states that provided $S$ satisfies NFLVR, this pair of spaces is in a perfect polar relation:

$$
\begin{aligned}
\mathcal{C} & =(\mathcal{D})^{0} \equiv\left\{g \in L^{0} \mid E g h \leq 1 \text { for all } h \in \mathcal{D}\right\} \\
\mathcal{D} & =(\mathcal{C})^{0} \equiv\left\{h \in L^{0} \mid E g h \leq 1 \text { for all } g \in \mathcal{C}\right\}
\end{aligned}
$$

Proof of Proposition: Note that $(A)$ is equivalent to $U^{\prime}\left(x^{*} X_{T}^{*}\right)=y^{*} Y_{T}^{*}$. Then the concavity of $U$ implies that for any $X \in \mathcal{X}$,

$E U\left(x^{*} X_{T}\right)-E U\left(x^{*} X_{T}^{*}\right) \leq x^{*} E\left(U^{\prime}\left(x^{*} X_{T}^{*}\right)\left[X_{T}-X_{T}^{*}\right]\right)=x^{*} y^{*} E\left(Y_{T}^{*}\left(X_{T}-X_{T}^{*}\right)\right)$.

But $Y_{T}^{*} \in \mathcal{D}$ and hence (5) implies that $E Y_{T}^{*} X_{T} \leq 1$. This proves that $\left(x^{*}, X^{*}\right)$ solves the primal problem.

The convexity of $V$ implies that for any $Y \in \mathcal{Y}$,

$E V\left(y^{*} Y_{T}\right)-E V\left(y^{*} Y_{T}^{*}\right) \geq y^{*} E\left(V^{\prime}\left(y^{*} Y_{T}^{*}\right)\left(Y_{T}-Y_{T}^{*}\right)\right)=x^{*} y^{*} E\left(X_{T}^{*}\left(Y_{T}^{*}-Y_{T}\right)\right)$.

But $X_{T}^{*} \in \mathcal{C}$ and hence (4) implies that $E X_{T}^{*} Y_{T} \leq 1$. This proves that $\left(y^{*}, Y^{*}\right)$ solves the dual problem.

To prove (3), note that $(A)$ implies

$$
U\left(x^{*} X_{T}^{*}\right)=V\left(y^{*} Y_{T}^{*}\right)+x^{*} y^{*} X_{T}^{*} Y_{T}^{*}
$$

Using condition (B),

$$
u\left(x^{*}\right)=E U\left(x^{*} X_{T}^{*}\right)=E V\left(y^{*} Y_{T}^{*}\right)+x^{*} y^{*}=v\left(y^{*}\right)+x^{*} y^{*} .
$$

But

$$
\begin{aligned}
u\left(x^{*}\right) & \leq \inf _{Y \in \mathcal{Y}} \sup _{g \in L^{0} ; E g Y_{T} \leq 1} E U\left(x^{*} g\right) \\
& \leq \inf _{y>0, Y \in \mathcal{Y}} \sup _{g \in L^{0}} E U\left(x^{*} g\right)-x^{*} y\left(E Y_{T} g-1\right) \\
& =\inf _{y>0, Y \in \mathcal{Y}} E V\left(y Y_{T}\right)+x^{*} y \\
& =\inf _{y>0} v(y)+x^{*} y \leq v\left(y^{*}\right)+x^{*} y^{*} .
\end{aligned}
$$

Thus these relations hold with equality, and (3) is proved.

\section{Log optimal portfolios in exponential Lévy markets}

We now consider an agent with the utility functions $U(x)=\log x, V(y)=$ $-\log y-1$ investing in a $1+1$ dimensional exponential Lévy model $\left(B_{t}, S_{t}\right)$ 
with $B_{t}=1$. The discounted risky asset is taken to be $S_{t}=S_{0} \mathcal{E}(L)_{t}$ where $L_{t}$ is the Lévy process with characteristic triple $\left(b, \sigma^{2}, \nu\right)$. That is, $S_{t}$ satisfies the stochastic differential equation

$$
\begin{aligned}
& d S_{t}=S_{t^{-}} d L_{t} \\
& d L_{t}=b d t+\sigma d W_{t}+\int_{-1}^{\infty}\left[z I_{l e}(z)[\mu-\nu](d s, d z)+z I_{>}(z) \mu(d s, d z)\right]
\end{aligned}
$$

where $\mu$ is the random measure with compensator $\nu, \mathrm{I}_{\leq}(z)=1-I_{>}(z)$ is the characteristic function of the interval $[-1,1], b, \sigma$ are constants and the Lévy measure $\nu(d z)$ is supported on the half infinite interval $(-1, \infty)$ and is such that $1 \wedge z^{2}$ is $\nu$-integrable. The support condition implies that the log of $S$ exhibits unpredictable positive and negative jumps which are unbounded.

In this market, the admissible processes are

$$
\mathcal{X}_{0}(x)=\left\{X_{t}=x+H \cdot S_{t} \mid 0 \leq H_{t} \leq S_{t}^{-1} X_{t}\right\}
$$

The positivity of $H$ reflects that the stock may jump unpredictably to an arbitrarily large value, hence shortselling will always lead to wealth which is unbounded from below with finite probability. That is, the conditional probability $P\left(X_{t^{+}}<-K \mid S_{t^{+}} \neq S_{t^{-}}\right)>0$ for any value $K>0$ if $H_{t^{-}}<0$. The upper bound on $H$ reflects that the bank has imposed a no borrowing constraint because the risky stock may jump unpredictably to an arbitrarily small positive value, meaning $P\left(X_{t^{+}}<0 \mid S_{t^{+}} \neq S_{t^{-}}\right)>0$ if $H_{t^{-}}>S_{t^{-}}^{-1} X_{t^{-}}$.

In view of the no borrowing, no shortselling constraints, the dual processes have the following representation:

$$
\mathcal{Y}=\left\{Y_{t} \geq 0 \mid Y_{0}=1 ; Y_{t}, S_{t} Y_{t} \text { are both supermartingales }\right\} .
$$

The optimal solution can be expressed in terms of

$$
\phi^{*}=\operatorname{argmax}_{\phi \in[0,1]} F(\phi),
$$

the maximizer over the interval $[0,1]$ of the concave function

$$
F(\phi)=b \phi-\sigma^{2} \phi^{2} / 2+\int\left[\log (1+\phi z)-\phi z I_{\leq}\right] \nu(d z) .
$$

Proposition 3.1 For each $x^{*}>0$, the quadruple $\left(x^{*}, y^{*}=1 / x^{*}, X^{*}=\right.$ $\left.\mathcal{E}\left(\phi^{*} L\right), Y^{*}=\mathcal{E}(M)\right)$ solves the primal/dual problem of Proposition 2.1 for utility $U(x)=\log x$ where

$M_{t}=-\int_{0}^{t} \phi^{*} \sigma d W_{s}+\int_{0}^{t} \int_{-1}^{\infty}\left[\left(1+\phi^{*} z\right)^{-1}-1\right][\mu-\nu](d s, d z)-\int_{0}^{t} \phi^{*} F^{\prime}\left(\phi^{*}\right) d s$

and $\phi^{*}$ is the constant defined by: 
(a) If $F^{\prime}(0)<0, \phi^{*}=0$;

(b) If $F^{\prime}(1)>0, \phi^{*}=1$;

(c) If $F^{\prime}(1) \leq 0 \leq F^{\prime}(0), \phi^{*} \in[0,1]$ is the unique root of $F^{\prime}(\phi)=0$.

Furthermore, the value functions are given by

$$
u(x)=\log x+K^{*} T, \quad v(y)=-\log y-1+K^{*} T
$$

where $K^{*}=F\left(\phi^{*}\right)$.

Remark: The above result can easily be extended to the general CRRA utility $U(x)=x^{p} / p, p \in(-\infty, 1) \backslash\{0\}$, see [3].

Proof: First note that $M_{t}$ is always a well-defined Lévy process: one need only check that $\int_{-1}^{-1 / 2}(1+z)^{-1} \nu(d z)<\infty$ when $F^{\prime}(1)>0$. To verify that $X^{*} \in \mathcal{X}$, note that $X_{t}^{*}=\mathcal{E}\left(\phi^{*} L\right)_{t}$ satisfies the stochastic differential equation

$$
d X_{t}^{*}=\left(\phi^{*} X^{*} / S\right)_{t^{-}} d S_{t}
$$

which means that $X^{*}$ has the admissible portfolio weight $H^{*}=\phi^{*} X^{*} / S$. To verify that $Y^{*} \in \mathcal{Y}$ first note that since $M_{t}$ is a supermartingale so is $Y_{t}^{*}=\mathcal{E}(M)_{t}$. Now $Y^{*} S=S_{0} \mathcal{E}(M) \mathcal{E}(L)=S_{0} \mathcal{E}(M+L+[M, L])$ and we calculate

$$
\begin{aligned}
& d(M+L+[M, L])_{t}=\text { martingale }+ \\
& \quad\left(-\phi^{*} F^{\prime}\left(\phi^{*}\right)+b-\sigma^{2} \phi^{*}+\int\left[z\left(\left(1+\phi^{*} z\right)^{-1}-I_{\leq}(z)\right)\right] \nu(d z)\right) d t .
\end{aligned}
$$

If $F^{\prime}(0) \geq 0$ then this is a martingale, but if $F^{\prime}(0)<0$ it is a strict supermartingale because the drift is strictly negative.

Since $-V^{\prime}\left(y^{*} Y_{T}^{*}\right)=\left(y^{*} Y_{T}^{*}\right)^{-1}$, both the conditions $(A),(B)$ of Proposition 2.1 follow by observing that $X_{t}^{*} Y_{t}^{*}=\mathcal{E}\left(\phi^{*} L+M+\phi^{*}[L, M]\right)_{t}=1$, which can be shown as in the previous calculation.

To calculate $u\left(x^{*}\right)=E \log \left(x^{*} X_{T}^{*}\right)$, we use Itô's formula to show

$$
\log \left(x^{*} X_{T}^{*}\right)=\log x^{*}+\int_{0}^{T} F\left(\phi^{*}\right) d t+\text { martingale }
$$

from which we deduce the result. 


\section{Discussion:}

The different cases in the above example illustrate what can happen when a security is overpriced in this market. Thus, in case (a) the mean rate of return of the stock is negative, which means the investor is disinclined to invest in it. Moreover, since any shortselling leads to an expected utility $-\infty$, the investor will hold exactly zero stock. It is then not surprising that the stock price is a strict supermartingale under the pricing measure $Q$ with $d Q / d P=Y_{T}^{*}$ : the fair price $E Y_{T}^{*} S_{T}$ is strictly less than the actual price $S_{0}$, meaning the market has overpriced the stock. There is no arbitrage because of the shortselling constraint.

Conversely, in case (b) the pricing measure $Q$ is not a probability measure. This is just to say that the fair price of the bond $E Y_{T}^{*}$ is strictly less than the actual market price 1 . Thus in case (b), the investor just holds stock: he cannot borrow because of the no borrowing constraint. In this case, $Y_{t}^{*}$ is not a martingale, but rather the "pricing kernel" defined to be inverse of the "natural numeraire" $X_{t}^{*}=S_{t}^{1} / S_{0}^{1}$.

In general, it is perfectly possible that an asset $S^{i}$ is overpriced. If shortselling is admissible, then the agent will include a negative number of units of $S^{i}$ in the optimal portfolio. Under the pricing measure, the discounted process $S^{i}$ will be a martingale. However, if shortselling is not admissible, then the optimal portfolio contains exactly zero units and $S^{i}$ will be a strict $Q$-supermartingale.

\section{References}

[1] D. Becherer. Rational Hedging and Valuation with Utility-based Preferences. PhD thesis, Technischen Universitat Berlin, 2001.

[2] F. Bellini and M. Frittelli. On the existence of minimax martingale measures. Math. Finance, 12(1):1-21, 2002.

[3] T. Choulli and T. R. Hurd. The role of hellinger processes in mathematical finance. Entropy, 3:150-161, 2001.

[4] F. Delbaen, P. Grandits, T. Rheinländer, D. Samperi, M. Schweizer, and C. Stricker. Exponential hedging and entropic penalties. Math. Finance, 12(2):99-123, 2002.

[5] F. Delbaen and W. Schachermayer. The fundamental theorem of asset pricing for unbounded stochastic processes. Math. Ann., 312(2):215-250, 1998. 
[6] M. Frittelli. Optimal solutions to utility maximization and to the dual problem. Preprint, 2002.

[7] T. Goll and J. Kallsen. Optimal portfolios for logarithmic utility. Stochastic Process. Appl., 89(1):31-48, 2000.

[8] P. Grandits. On martingale measures for stochastic processes with independent increments. Teor. Veroyatnost. i Primenen., 44(1):87-100, 1999.

[9] J. Jacod and A. N. Shiryaev. Limit theorems for stochastic processes. Springer-Verlag, Berlin, 1987.

[10] J. Kallsen. Optimal portfolios for exponential Lévy processes. Math. Methods Oper. Res., 51(3):357-374, 2000.

[11] I. Karatzas and S. E. Shreve. Methods of mathematical finance, volume 39 of Applications of Mathematics. Springer-Verlag, New York, 1998.

[12] D. Kramkov and W. Schachermayer. The asymptotic elasticity of utility functions and optimal investment in incomplete markets. Ann. Appl. Probab., 9(3):904-950, 1999.

[13] R. C. Merton. Optimum consumption and portfolio rules in a continuous-time model. J. Econom. Theory, 3:373-413, 1971.

[14] M. P. Owen. Utility based optimal hedging in incomplete markets. Ann. Appl. Probab., 12(2):691-709, 2002.

[15] W. Schachermayer. Optimal investment in incomplete financial markets. In H. Geman et al., editors, Mathematical Finance: Bachelier Congress 2000, pages 427-462, 2001. 